\title{
Nicotinic Acetylcholine Receptor-Mediated Signaling Pathways in Pluripotent Stem Cells
}

\author{
Takashi Yazawa ${ }^{1 *}$, Junsuke Uwada', Yoshitaka Imamichi², Daisuke Mikami ${ }^{3}$, Takahiro Sato ${ }^{4}$, \\ Rafiqul Islam Khan ${ }^{1,5}$, Mohammad Sayful Islam ${ }^{1,6}$ and Takanobu Taniguchi ${ }^{1}$ \\ ${ }^{1}$ Department of Biochemistry, Asahikawa Medical University, Japan
}

${ }^{2}$ Department of Pharmacology, Asahikawa Medical University, Japan

${ }^{3}$ Department of Nephrology, University of Fukui, Japan

${ }^{4}$ Institute of Life Science, Kurume University, Japan

${ }^{5}$ Department of Pharmacy, University of Rajshahi, Bangladesh

${ }^{6}$ Department of Pharmacy, Mawalana Bhashani Science and Technology University, Bangladesh

*Corresponding author: Takashi Yazawa, Department of Biochemistry, Asahikawa Medical University, Midorigaoka Higashi 2-1-1-1, Asahikawa, Hokkaido 078-8510, Japan.

To Cite This Article: Takashi Yazawa, Nicotinic Acetylcholine Receptor-Mediated Signaling Pathways in Pluripotent Stem Cells. Am J Biomed Sci \& Res. 2019 - 6(5). AJBSR.MS.ID.001061. DOI: 10.34297/AJBSR.2019.06.001061.

Received: 眥 December 3, 2019; Published: 制 December 11, 2019

\section{Mini Review}

Electronic cigarettes (E-cigarettes) are battery-operated devices that transport a nicotine- containing aerosol or vapor by heating the liquid. The liquid usually contains nicotine, propylene glycol or glycerol, Acetylcholine receptors (AChRs) are membrane receptors that bind to the neurotransmitter acetylcholine. They are classified into two distinct subtypes, nicotinic AChRs (nAChRs) and muscarinic AChRs (mAChRs). nAChRs belong to the Cys-loop family of pentameric ligand-gated ion channels. They consist of seventeen subunits, various $\alpha$ (CHRNA1-10, but CHRNA8 is avian specific)

and $\beta$ (CHRNB1-4) subunits with $\delta$ (CHRND), $\quad \gamma$ (CHRNG) and $\varepsilon$ (CHRNE) subunits [1,2]. These subunits can be divided into neuronal-type (CHRNA2-10 and CHRNB2-4) and muscle-type (CHRNA1, CHRNB1, CHRND, CHRNG, CHRNE). Although they form various heteromeric pentamers by combination of any $\alpha$ subunits and other subunits, some $\alpha$ subunits (CHRNA7 CHRNA8 and CHRNA9) function ashomomeric pentamers. Upon binding to ligands, pentameric receptors undergo conformational changes to open a central pore, causing the influx of extracellular ions and various cellular responses.

\begin{tabular}{|c|c|c|c|}
\hline \multirow{2}{*}{ Table 1: Expression of CHRNA/Chrna and CHRNB/Chrnb genes in human and murine pluripotent stem cells. } \\
\hline & $\begin{array}{c}\text { human ESCs (BG02, WA09) } \\
{[\mathbf{1 0 ]}}\end{array}$ & $\begin{array}{c}\text { murine ESCs (CGR8) } \\
\text { [11] }\end{array}$ & $\begin{array}{c}\text { murine IPSCs (iPS-MEF-Ng-20D-17) [7] (unpub- } \\
\text { lished data) }\end{array}$ \\
\hline CHRNA1/Chrna1 & + & - & + \\
\hline CHRNA2/Chrna2 & - & + & + \\
\hline CHRNA3/Chrna3 & + & + & + \\
\hline CHRNA4/Chrna4 & - & - & ++ \\
\hline CHRNA5/Chrna5 & ++ & - & + \\
\hline CHRNA6/Chrna6 & - & + & + \\
\hline CHRNA7/Chrna7 & - & + & + \\
\hline CHRNA9/Chrna9 & + & + & + \\
\hline CHRNA10/Chrna10 & + & + & + \\
\hline CHRNB1/Chrnb1 & + & + & + \\
\hline CHRNB2/Chrnb2 & - & + & + \\
\hline CHRNB3/Chrnb3 & + & + & + \\
\hline CHRNB4/Chrnb4 & - & + & + \\
\hline
\end{tabular}

Note: Table Abbreviations: ESCs: Embryonic Stem Cells; iPSCs: induced-Pluripotent Stem Cells. 
Consistent with the findings for classification of receptor genes, nAChR-mediated signaling pathways play important roles in neuron and muscle [3]. In addition to these tissues, nAChR genes are also expressed in various non-neuronal tissues and cell types $[4,5]$. Pluripotent stem cells (PSCs), so-called embryonic stem cells (ESCs) and induced pluripotent stem cells (iPSCs), represent one of such cell types. ESCs are derived from the inner cell mass of preimplantation embryo, whereas iPSCs are generated through somatic cell reprograming by the overexpression of defined transcription factors (Yamanaka factors) [6]. These are expected to be an ideal source for novel regenerative medicine $[7,8]$. PSCs have unique characteristics that undergo unlimited self-renewal and retain pluripotency to differentiate into any cell types. Various signaling pathways are involved in maintaining the delicate balance between self-renewal and differentiation in PSCs [9]. They are also important for reprograming of somatic cells to establish iPSCs. Elucidation of these pathways is essential for the clinical applications of these cells. Both human and murine PSCs express various CHRNA/Chrna and CHRNB/Chrnb genes [10,11] (Table 1). In addition, expression pattern of nAChR genes dynamically fluctuates during differentiation of PSCs into various cell lineages, including neuronal cells and myocytes. These finding indicate that nAChR-mediated signaling pathways play important roles in PSCs.

Infact, nAChR-mediated signalingpathways affectdifferentiation of PSCs. Triggering of nAChRs expressed in human ESCs-derived embryoid bodies by nicotine resulted in activation of MAPK and shifts of spontaneous differentiation toward hemangioblast [12]. In contrast, Gue et al. reported deleterious effects of nicotine on human ESCs-derived various lineages, including cardiomyoctes, by using single cell RNA-sequencing [13]. Consistent with the results in human ESCs, nAChR-signaling pathways inhibit differentiation of mESCs into cardiomyocytes by suppressing cardiac genes via DNA methylation [14]. Doubling time is reduced by nAChRmediated signaling via downregulation of $\mathrm{N}$-myc expression during differentiation of primate ESCs into fibroblasts [15]. Taken together, it is conceivable that effects of nAChR-mediated signaling pathways on differentiation of PSCs are dependent on the cell lineages.

In addition to the differentiation processes, nAChRmediated singling pathways likely contribute to self-renewal and establishment of PSCs. Because murine and human ESCs express choline acetyltransferase and synthesize ACh [12,16], nAChRmediated signaling pathways should be constitutively activated in PSCs. In support of this hypothesis, exogenous Ach and nicotine additions affect the proliferation and survival of PSCs via nAChRs. Nicotine increases DNA synthesis via some Chrna pathways in murine iPSCs $[17,18]$. In murine ESCs, high doses of ACh and nicotine reduce apoptosis, but they inhibit proliferation [19]. In contrast, it is unknown whether nAChR-mediated signaling pathways are involved in the undifferentiated status, pluripotency and reprograming process. To achieve a comprehensive understanding of the roles of nAChR-mediated signaling pathways in PSCs, it is necessary in future studies to investigate the functions of each CHRNA/Chrna and CHRNB/Chrnb gene that are expressed in PSCs. It is also important to evaluate nAChR-mediated signaling pathways that are activated not only by exogenous ligands, but also by endogenous ACh. Such studies will provide essential insights to ensure the use of PSCs in future regenerative medicine.

\section{Acknowledgments}

We thank to Ms. S. Tsunoda for administrative assistance. This study was performed under the cooperative research program of Institute of Nature and Environmental Technology, Kanazawa University (Accept No.19030) in 2019. This work was supported in part by JSPS Grant Number 19K09794 (Grant-in-Aid for Scientific Research (C)), 18K15971 (Grant-in-Aid for Scientific Research (C)), 17K11214 (Grant-in-Aid for Scientific Research (C)) and 19K07117 (Grant-in-Aid for Scientific Research (C)) granted by Japan Society for the Promotion of Science, the Smoking Research Foundation of Japan and by the Grant of National Center for Child Health and Development.

\section{Conflict of Interest}

All authors have nothing to disclose.

\section{References}

1. Hone AJ, McIntosh JM (2018) Nicotinic acetylcholine receptors in neuropathic and 90 inflammatory pain. FEBS Lett 592(7): 1045-1062.

2. Liu W, Li MD (2018) Insights Into Nicotinic Receptor Signaling in Nicotine Addiction: Implications for Prevention and Treatment. Curr Neuropharmacol 16(4): 350-370.

3. Hogg RC, Raggenbass M, Bertrand D (2003) Nicotinic acetylcholine receptors: from structure to brain function. Rev Physiol Biochem Pharmacol. 147: 1-46.

4. Zoli M, Pucci S, Vilella A, Gotti C (2018) Neuronal and Extraneuronal Nicotinic Acetylcholine Receptors. Curr Neuropharmacol 16(4): 338349 .

5. Fujii T, Mashimo M, Moriwaki Y, Misawa H, Ono S, et al. (2017) Physiological functions of the cholinergic system in immune cells. J Pharmacol Sci 134(1): 1-21.

6. Shi Y, Inoue H, Wu JC, Yamanaka S (2017) Induced pluripotent stem cell technology: a decade of progress. Nat Rev Drug Discov 16(2): 115-130.

7. Yazawa T, Kawabe S, Inaoka Y, Okada R, Mizutani T, et al. (2011) Differentiation of mesenchymal stem cells and embryonic stem cells into steroidogenic cells using steroidogenic factor-1 and liver receptor homolog-1. Mol Cell Endocrinol 336(1-2): 127-132.

8. Yazawa T, Imamichi Y, Miyamoto K, Khan MR, Uwada J, et al. (2016) Induction of steroidogenic cells from adult stem cells and pluripotent stem cells [Review]. Endocr J 63(11): 943-951.

9. Niwa H (2007) How is pluripotency determined and maintained? Development 134(4): 635-646.

10. Krishnamoorthy M, Gerwe BA, Scharer CD, Heimburg Molinaro J, Gregory F, et al. (2010) Low ethanol concentration alters CHRNA5 RNA levels during early human development. Reprod Toxicol 30(3): 489-492.

11. Kaltwasser S, Schmitz L, Michel Schmidt R, Anspach L, Kirkpatrick CJ, et al. (2015) Murine embryonic stem cell line CGR8 expresses all subtypes of muscarinic receptors and multiple nicotinic receptor subunits: Down- 
regulation of alpha4- and beta4- subunits during early differentiation. Int Immunopharmacol 29(1): 110-114.

12. Serobyan N, Jagannathan S, Orlovskaya I, Schraufstatter I, Skok M, et al. (2007) The cholinergic system is involved in regulation of the development of the hematopoietic system. Life Sci 80(24-25): 23522360

13. Mao X, An Q, Xi H, Yang XJ, Zhang X, et al. (2019) Single-Cell RNA Sequencing of hESC-Derived 3D Retinal Organoids Reveals Novel Genes Regulating RPC Commitment in Early Human Retinogenesis. Stem Cell Reports 13(4): 747-760.

14. Jiang XY, Feng YL, Ye LT, Li XH, Feng J, et al. (2017) Inhibition of Gata4 and Tbx5 by Nicotine-Mediated DNA Methylation in Myocardial Differentiation. Stem Cell Reports 8(2): 290-304.

15. Ben Yehudah A, Campanaro BM, Wakefield LM, Kinney TN, Brekosky J, et al. (2013) Nicotine exposure during differentiation causes inhibition of $\mathrm{N}$-myc expression. Respir Res 14: 119.
16. Paraoanu LE, Steinert G, Koehler A, Wessler I, Layer PG (2007) Expression and possible functions of the cholinergic system in a murine embryonic stem cell line. Life Sci 80(24-25): 2375-2379.

17. Ishizuka T, Goshima H, Ozawa A, Watanabe Y (2012) Effect of nicotine on the proliferation and differentiation of mouse induced pluripotent stem cells. Curr Med Chem 19(30): 5164-5169.

18. Ishizuka T, Ozawa A, Goshima H, Watanabe Y (2012) Involvement of nicotinic acetylcholine receptor in the proliferation of mouse induced pluripotent stem cells. Life Sci 90(17-18): 637-648.

19. Landgraf D, Barth M, Layer PG, Sperling LE (2010) Acetylcholine as a possible signaling molecule in embryonic stem cells: studies on survival, proliferation and death. Chem Biol Interact 187(1-3): 115-119. 